\title{
Linear differential algebraic equations of index 1 and their adjoint equations
}

\author{
Katalin Balla * \\ Computer and Automation Research Institute, \\ Hungarian Academy of Sciences \\ Budapest, Hungary \\ H-1518 Budapest P.O.Box 63 \\ Balla@sztaki.hu \\ Roswitha März \\ Humboldt University \\ D-10099 Berlin, Germany \\ Unter den Linden 6 \\ iam@mathematik.hu-berlin.de
}

\begin{abstract}
For linear differential algebraic equations of tractability index 1 the notion of the adjoint equation is analysed in full detail. Its solvability is shown at the lowest possible smoothness. The fundamental matrices of both equations are defined and their relationships are characterized.

Keywords: linear differential algebraic equations of index 1, adjoint equation, solvability, fundamental matrices.
\end{abstract}

Mathematics Subject Classification: 34A09, 34A30

*The work of this author was supported by the Hungarian National Science Foundation, Grant No. T029572. 


\section{Introduction}

In the classical theory of linear homogeneous ordinary differential equations (ODEs), the adjoint equations are derived as those which are satisfied by the inverse adjoint matrices to the fundamental matrices of solutions [5]. Due to Lagrange identity, they play a central role when one characterizes the linear subspaces of solutions of the original ODEs. This fact explains their importance in the solution of boundary value problems for linear ODEs [1].

The monograph [6] was devoted to differential algebraic equations (DAEs). A number of problems for which the similarity or dissimilarity to regular ODEs were essential was highlighted there. Some questions were raised later. In papers $[2,3]$, we have proven that for the linear homogeneous differential algebraic equations of index 1 with differentiable coefficients there exist differential algebraic equations - we called them adjoint DAEs - such that any pair of solutions of an original DAE and that of its adjoint equation satisfies an identity which may be considered as an analogue of the Lagrange identity. Moreover, we succeeded to prove that this identity appears in the characterization of linear subspaces of solutions of the original DAEs, as well. Due to differentiability, we could also apply a theorem of reduction type and we were able to show that the adjoint equation to the regular ODE obtained by the reduction naturally leads to the adjoint equation for the DAE.

The aim of this paper is twofold. First, we relax the differentiability of the coefficients of the DAE. We show that a pendant of the original DAE - we call it adjoint equation - is solvable at the smoothness appearing in the definition which assures the solvability of the original DAE of index 1. This condition is weaker than differentiability of the coefficients. Note that without differentiability, the adjoint equation is not a DAE in standard form. Consequently, neither an index can be assigned directly. Therefore we give a proper definition. At this low smoothness, neither the theorems of reduction type work. Secondly, we show that one can arrive to the homogeneous adjoint equation by construction of an equation which is satisfied by a properly transformed reflexive generalized inverse of a fundamental matrix of the original DAE. The statement is as similar to the regular ODE case as possible. Meanwhile, we clarify the relationships between the different fundamental matrices of a DAE. This question was not addressed in [6]. We discuss the notion of the fundamental matrix for the adjoint equation and its properties, too. We obtain the analogue of Lagrange identity at the lowest smoothness 
of coefficients.

In contrast to $[2,3]$ we do not assume that the projectors appearing in our analysis are orthogonal. In the previous works, real DAEs were considered. The extension of the study to the complex case does not require extra efforts, so we describe the results for the complex case.

\section{The DAE of index 1 and its adjoint}

Let $A: \mathcal{I} \rightarrow L\left(\mathbb{C}^{m}, \mathbb{C}^{m}\right), \mathcal{I} \subseteq \mathbb{R}$. Let $Q: \mathcal{I} \rightarrow L\left(\mathbb{C}^{m}, \mathbb{C}^{m}\right)$ be an arbitrary projector onto $\operatorname{Ker} A$, i.e., for all $x \in \mathcal{I}, A(x) Q(x)=0 \in L\left(\mathbb{C}^{m}, \mathbb{C}^{m}\right)$. Let us denote $P=I-Q$, where $I$ is the identity operator: $I y=y$ for all $y: \mathcal{I} \rightarrow \mathbb{C}^{m}$.

Let $P$ be as above. If

$$
y: \mathcal{I} \rightarrow \mathbb{C}^{m}, \quad P y \in C^{1}\left(\mathcal{I}, \mathbb{C}^{m}\right) \quad \text { and } \quad P \in C^{1}\left(\mathcal{I}, L\left(\mathbb{C}^{m}, \mathbb{C}^{m}\right)\right),
$$

then $A(P y)^{\prime}-A P^{\prime} y$ is well defined. We can check $A(P y)^{\prime}-A P^{\prime} y$ is independent of the special choice of projector $P$.

Let $B: \mathcal{I} \rightarrow L\left(\mathbb{C}^{m}, \mathbb{C}^{m}\right)$. Then, for all $y: \mathcal{I} \rightarrow \mathbb{C}^{m}$ and all projectors $Q \in C^{1}\left(\mathcal{I}, L\left(\mathbb{C}^{m}, \mathbb{C}^{m}\right)\right)$ onto $\operatorname{Ker} A$ such that $P y \in C^{1}\left(\mathcal{I}, \mathbb{C}^{m}\right)$, the operator $\mathcal{L}$ given by

$$
\mathcal{L} y:=A(P y)^{\prime}+\left(B-A P^{\prime}\right) y
$$

is well defined and $\mathcal{L} y \in\left\{\tilde{y}: \mathcal{I} \rightarrow \mathbb{C}^{m}\right\}$. Moreover, $\mathcal{L} y$ does not depend on specific choice of $Q$. In order to make an accent on this independence, i.e., on the dependence of $\mathcal{L}$ on $A$ and $B$, only, we write sometimes $\mathcal{L} y=A y^{\prime}+B y$. Notice that the right hand side is meaningful and coincides with the left hand side provided $y \in C^{1}\left(\mathcal{I}, \mathbb{C}^{m}\right)$.

We may also verify that $P_{1} y \in C^{1}\left(\mathcal{I}, \mathbb{C}^{m}\right)$ and $P_{1}, P_{2} \in C^{1}\left(\mathcal{I}, L\left(\mathbb{C}^{m}, \mathbb{C}^{m}\right)\right)$ involve $P_{2} y \in C^{1}\left(\mathcal{I}, \mathbb{C}^{m}\right)$ for all $y: \mathcal{I} \rightarrow \mathbb{C}^{m}$, as well. Thus, the function space $\left\{y: \mathcal{I} \rightarrow \mathbb{C}^{m}, P y \in C^{1}\left(\mathcal{I}, \mathbb{C}^{m}\right)\right\}$ where $Q \in C^{1}\left(\mathcal{I}, L\left(\mathbb{C}^{m}, \mathbb{C}^{m}\right)\right)$ is an arbitrary projector onto $\operatorname{Ker} A$ does not depend on the special choice of $Q$. So is with the function space $\left\{y \in C\left(\mathcal{I}, \mathbb{C}^{m}\right): P y \in C^{1}\left(\mathcal{I}, \mathbb{C}^{m}\right)\right\}$. Thus, the latter function space is defined by $\operatorname{Ker} A$ uniquely, we denote it by $C_{A}^{1}\left(\mathcal{I}, \mathbb{C}^{m}\right)$.

When a fixed basis is given in $\mathbb{C}^{m}$, we speak about the elements of $\mathbb{C}^{m}$ as column vectors, and, correspondingly, the elements of $L\left(\mathbb{C}^{m}, \mathbb{C}^{m}\right)$ are matrices.

Our basic assumptions will be 
(T1) $\operatorname{dim} \operatorname{Im} A(x) \equiv r<m$ for all $x \in \mathcal{I}$,

(T2) the matrix pencil $\{A(x), B(x)\}$ is regular and ind $\{A(x), B(x)\}=1$, for all $x \in \mathcal{I}{ }^{1}$ and

(T3) there exists a projector $Q \in C^{1}\left(\mathcal{I}, L\left(\mathbb{C}^{m}, \mathbb{C}^{m}\right)\right)$ onto $\operatorname{Ker} A$.

With these assumptions, equation

$$
\mathcal{L} y=f
$$

is called transferable DAE, or, equivalently, DAE of tractability index 1. Let

$$
A, B \in C\left(\mathcal{I}, L\left(\mathbb{C}^{m}, \mathbb{C}^{m}\right)\right), \quad f \in C\left(\mathcal{I}, \mathbb{C}^{m}\right) .
$$

A function $y \in C_{A}^{1}\left(\mathcal{I}, \mathbb{C}^{m}\right)$ is called a solution of (2) if it turns equation (2) into identity. For the above, in more general setting, see \&1.2.1 and \&1.2.2 in the basic book [6] or the survey paper [8].

In the following we use notations $B_{0}:=B-A P^{\prime}, A_{1}=A+B_{0} Q$, observing that by Theorem 13, Appendix A in [6], $A_{1}$ is invertible provided (T2) holds.

The state variable form is the decomposition of (2) into the inherent regular ordinary differential equation

$$
\mathcal{L}_{s} P y=P A_{1}^{-1} f
$$

and the algebraic equation

$$
Q_{s} y=Q A_{1}^{-1} f
$$

where

$$
\mathcal{L}_{s} z:=z^{\prime}+\left(P A_{1}^{-1} B_{0}-P^{\prime}\right) z, \quad Q_{s} z:=Q z+Q A_{1}^{-1} B P z .
$$

This splitting is a result of rearrangement of the terms in (2) followed by the application of $A_{1}^{-1}$ to (2). Due to $Q_{s}^{2}=Q_{s}, Q_{s}$ turns to be a projector. We will characterize this projector in detail later. At the moment we recall from [6], Appendix A, Lemma 14 - where real coefficients are considered - that $Q_{s}(x)$ projects onto Ker $A(x)$ along $S(x)=\left\{v \in \mathbb{C}^{m}: B(x) v \in \operatorname{Im} A(x)\right\}$. We will recover this fact anew in a different context below.

\footnotetext{
${ }^{1}$ for definition of the index of a matrix pencil see e. g. Appendix A in [6] or [8]
} 
$\mathcal{L}_{s}$ is well-defined for $z \in C^{1}\left(\mathcal{I}, \mathbb{C}^{m}\right)$ and the initial value problem

$$
\mathcal{L}_{s} z=g, \quad z(\hat{x})=\hat{z}, \quad \hat{x} \in \mathcal{I}, \quad \hat{z} \in \mathbb{C}^{m} \quad g \in C\left(\mathcal{I}, \mathbb{C}^{m}\right)
$$

is uniquely solvable in $C^{1}\left(\mathcal{I}, \mathbb{C}^{m}\right)$. Moreover, $z \in \operatorname{Im} P$ is valid provided that $\hat{z} \in \operatorname{Im} P(\hat{x}), g \in \operatorname{Im} P$ hold.

Let $\mathcal{L}_{s}^{*}$ be defined as the adjoint of $\mathcal{L}_{s}$,

$$
\mathcal{L}_{s}^{*} w:=w^{\prime}-\left(B_{0}^{*} A_{1}^{*-1} P^{*}-P^{* \prime}\right) w
$$

for $w \in C^{1}\left(\mathcal{I}, \mathbb{C}^{m}\right)$.

Then, again, the initial value problem

$$
\mathcal{L}_{s}^{*} w=h, \quad w(\hat{x})=\hat{w}, \quad \hat{x} \in \mathcal{I}, \quad \hat{w} \in \mathbb{C}^{m}, \quad h \in C\left(\mathcal{I}, \mathbb{C}^{m}\right)
$$

is uniquely solvable in $C^{1}\left(\mathcal{I}, \mathbb{C}^{m}\right)$. Meanwhile, $P^{*} w \in C^{1}\left(\mathcal{I}, \mathbb{C}^{m}\right)$, too, and

$$
\left(P^{*} w\right)^{\prime}-\left(P^{* \prime}+P^{*} B_{0}^{*} A_{1}^{*-1}\right) P^{*} w=P^{*} h, \quad P^{*}(\hat{x}) w(\hat{x})=P^{*}(\hat{x}) \hat{w} .
$$

Let $u:=A^{+*} P^{*} w$, where $A^{+}$is the Moore-Penrose inverse of $A$. Then, $u \in C\left(\mathcal{I}, \mathbb{C}^{m}\right)$ and

$$
A^{*} u=A^{*} A^{+*} P^{*} w=\left(A^{+} A\right)^{*} P^{*} w=P^{*} w \in C^{1}\left(\mathcal{I}, \mathbb{C}^{m}\right) .
$$

Noting that $P^{*} A_{1}^{*}=A^{*}, A^{*}=A^{*} A_{1}^{*-1} A^{*}, Q^{*} B_{0}^{*}=Q^{*} A_{1}^{*}$ and with

$$
P_{* s}:=A_{1}^{*-1} P^{*} A_{1}^{*}=A_{1}^{*-1} A^{*},
$$

one has

$$
\begin{array}{r}
P^{*} h=\left(A^{*} u\right)^{\prime}-\left(P^{* \prime}+P^{*} B_{0}^{*} A_{1}^{*-1}\right) A^{*} u \\
=\left(A^{*} u\right)^{\prime}-\left(P^{* \prime} A^{*}-Q^{*} B_{0}^{*} A_{1}^{*-1} A^{*}+B_{0}^{*} A_{1}^{*-1} A^{*}\right) u \\
=\left(A^{*} u\right)^{\prime}-\left(P^{* \prime} A^{*}-Q^{*} A_{1}^{*} A_{1}^{*-1} A^{*}+B_{0}^{*} A_{1}^{*-1} A^{*}\right) u \\
=\left(A^{*} u\right)^{\prime}-\left(P^{* \prime} A^{*} A_{1}^{*-1} A^{*}+B_{0}^{*} A_{1}^{*-1} A^{*}\right) u \\
=\left(A^{*} A_{1}^{*-1} A^{*} u\right)^{\prime}-\left(P^{* \prime} A^{*}+B_{0}^{*}\right) A_{1}^{*-1} A^{*} u \\
=\left[A^{*}\left(A_{1}^{*-1} A^{*} u\right)\right]^{\prime}-B^{*}\left(A_{1}^{*-1} A^{*} u\right)=\left[A^{*}\left(P_{* s} u\right)\right]^{\prime}-B^{*}\left(P_{* s} u\right) .
\end{array}
$$

We obtained that at least when $s=P^{*} h, h \in C\left(\mathcal{I}, \mathbb{C}^{m}\right)$, equation

$$
\mathcal{L}^{*} \phi=s, \quad \text { where } \quad \mathcal{L}^{*} \phi:=\left(A^{*} \phi\right)^{\prime}-B^{*} \phi,
$$


has at least one solution. Later we will call equation (13) adjoint equation of the DAE (2), but first we are going to approve this notation.

$P_{* s}$ is obviously a projector and $P_{* s} \in C\left(\mathcal{I}, L\left(\mathbb{C}^{m}, \mathbb{C}^{m}\right)\right)$. Let $Q_{* s}:=$ $I-P_{* s}$. We have

$$
Q_{* s}=A_{1}^{*-1} Q^{*} A_{1}^{*}
$$

By the formal definition, $\operatorname{dim} \operatorname{Im} P_{* s}=r, \operatorname{dim} \operatorname{Im} Q_{* s}=m-r$.

Lemma 1 The projector pair $P_{* s}, Q_{* s}$ is independent of the special choice of projector $P \in C^{1}\left(\mathcal{I}, L\left(\mathbb{C}^{m}, \mathbb{C}^{m}\right)\right)$.

Proof. When (T3) takes place, then, above the projector $P \in C^{1}(\mathcal{I}$, $\left.L\left(\mathbb{C}^{m}, \mathbb{C}^{m}\right)\right)$ indicated in (T3), the orthogonal projector $\breve{P}$ onto $\operatorname{Im} A^{*}$ is differentiable, as well. The latter is unique and $\breve{P}=A^{+} A=P^{+} P(A$ is not necessarily differentiable but $P$ is.). Therefore, it is sufficient to prove that for an arbitrary projector $P \in C^{1}\left(\mathcal{I}, L\left(\mathbb{C}^{m}, \mathbb{C}^{m}\right)\right)$ and the orthogonal projector $\breve{P}$, the projector $P_{* s}$ defined by $(12)$ and $\breve{P}_{* s}$ defined in a similar way, coincide, i.e.,

$$
P_{* s}=\breve{P}_{* s}
$$

Denote $\breve{A}_{1}:=A+\left(B-A \breve{P}^{\prime}\right) \breve{Q}$. Instead of showing (15), we check that for $R:=A^{*}-A_{1}^{*} \breve{A}_{1}^{*-1} A^{*}$, both $P^{*} R=0$ and $Q^{*} R=0$ hold. Then, we use $R=\left(P^{*}+Q^{*}\right) R$.

$$
\begin{array}{r}
P^{*} R=P^{*} A^{*}-\left(P^{*} A_{1}^{*}\right) \breve{A}_{1}^{*-1} A^{*}=A^{*}-A^{*} \breve{A}_{1}^{*-1} A^{*} \\
=A^{*}-\left(A^{*} \breve{A}_{1}^{*-1}\right) A^{*}=A^{*}-\breve{P}^{*} A^{*}=\breve{Q}^{*} A^{*}=(A \breve{Q})^{*}=0 .
\end{array}
$$

In order to verify that $Q^{*} R$ vanishes, we recall that orthogonality means $\breve{P}=\breve{P}^{*}, \breve{Q}=\breve{Q}^{*}$ and $\breve{P} Q=\left(A^{+} A\right) Q=A^{+}(A Q)=0$. Thus, $\breve{P}=\breve{P}(P+$ $Q)=\breve{P} P+\breve{P} Q=\breve{P} P$ and $Q=(\breve{P}+\breve{Q}) Q=\breve{P} Q+\breve{Q} Q=\breve{Q} Q$. Further, $P \breve{Q}=\left(A_{1}^{-1} A\right) \breve{Q}=A_{1}^{-1}(A \breve{Q})=0$. For $\breve{P}$ we also use the adjoint of the identities $A P^{\prime}=A P^{\prime} Q$ and $Q^{\prime}=-P^{\prime}$, which holds for arbitrary $P$. Now the required result comes from the following chain of equalities:

$$
\begin{array}{r}
Q^{*} R=Q^{*} A^{*}-Q^{*} A_{1}^{*} \breve{A}_{1}^{*-1} A^{*}=-Q^{*}\left(B^{*}-P^{* \prime} A^{*}\right) \breve{A}_{1}^{*-1} A^{*} \\
=-Q^{*} B^{*} \breve{A}_{1}^{*-1} A^{*}+Q^{*} P^{* \prime} A^{*} \breve{A}_{1}^{*-1} A^{*} \\
=-(\breve{Q} Q)^{*} B^{*} \breve{A}_{1}^{*-1} A^{*}+(\breve{Q} Q)^{*}(P \breve{P})^{* \prime} A^{*} \breve{A}_{1}^{*-1} A^{*} \\
=-Q^{*} \breve{Q}^{*} B^{*} \breve{A}_{1}^{*-1} A^{*}+\left[\left(Q^{*} \breve{Q} \breve{P} P^{*}\right)^{\prime}-\left(Q^{*} \breve{Q}\right)^{\prime} \breve{P} P^{*} A^{*} \breve{A}_{1}^{*-1} A^{*}\right.
\end{array}
$$




$$
\begin{array}{r}
=-Q^{*} \breve{Q}^{*} B^{*} \breve{A}_{1}^{*-1} A^{*}-\left(Q^{*} \breve{Q}\right)^{\prime} A^{*} \breve{A}_{1}^{*-1} A^{*} \\
=-Q^{*} \breve{Q}^{*} B^{*} \breve{A}_{1}^{*-1} A^{*}-\left[Q^{* \prime}\left(\breve{Q} A^{*}\right)-Q^{*} \breve{P}^{\prime} A^{*}\right] \breve{A}_{1}^{*-1} A^{*} \\
=-Q^{*}\left(\breve{Q} B^{*}-\breve{Q}^{\prime} \breve{P}^{\prime}\right) \breve{A}_{1}^{*-1} A^{*} \\
=-Q^{*} \breve{Q} \breve{A}_{1}^{*} \breve{A}_{1}^{*-1} A^{*}=-Q^{*} \breve{Q} A^{*}=0 .
\end{array}
$$

In this context it requires a slightly more preparation to verify that $Q_{s}$ and its complement $P_{s}:=I-Q_{s}$ are also projectors independent of $P$. Meanwhile, we get a representation of $Q_{s}, P_{s}$ as symmetric as (12) and (14) are. While by the help of the formal definition we could state only $\operatorname{Im} Q_{s} \subseteq \operatorname{Ker} A$, and the rearrangement

$$
\begin{array}{r}
Q_{s}:=Q+Q A_{1}^{-1} B P=Q A_{1}^{-1}\left(A_{1}+B P\right) \\
=Q A_{1}^{-1}\left[A+\left(B-A P^{\prime}\right) Q+B P\right]=Q\left(A_{1}^{-1} A\right)\left(I-P^{\prime}\right)+Q A_{1}^{-1} B \\
=Q P\left(I-P^{\prime}\right)+Q A_{1}^{-1} B=Q A_{1}^{-1} B
\end{array}
$$

does not make the value of $\operatorname{dim} \operatorname{Im} Q_{s}$ transparent, now we will be able to find this value as well.

For $A, B: \mathcal{I} \rightarrow L\left(\mathbb{C}^{m}, \mathbb{C}^{m}\right)$ denote

$$
A_{* 1}:=A^{*}-B_{0}^{*} Q_{*}=A^{*}-B^{*} Q_{*}
$$

where $Q_{*}$ be a projector onto $\operatorname{Ker} A^{*}$. The complement of $Q_{*}$ is denoted by $P_{*}, P_{*}:=I-Q_{*}$. For orthogonal projectors and functions derived by their use we keep notation ${ }^{\checkmark}$ and one has $\breve{P}_{*}=A A^{+}$. When (T2) holds then ind $\left\{A^{*}(x),-B^{*}(x)\right\}=1$ for all $x \in \mathcal{I}$ and $A_{* 1}$ is invertible due to Theorem 13, Appendix A in [6].

Remark 1 Since both $Q$ and $Q_{*}$ are arbitrary, $Q_{*}=Q^{*}, P_{*}=P^{*}$ cannot hold, in general. Neither $\breve{P}_{*}=\breve{P}^{*}$ is valid. Since $\breve{P}_{*}=A A^{+}, \breve{P}^{*}=\breve{P}=A^{+} A$, one obtains a necessary and sufficient condition for $\breve{P}_{*}=\breve{P}^{*}$, namely $\operatorname{Im} A=$ Im $A^{*}$ (or Im $A=\operatorname{Ker} A^{\perp}$ ). Clearly, for the selfadjoint matrices this holds true.

Neither can one claim that for a given $Q$ there exists $Q_{*}$ such that $Q_{*}=Q^{*}$ would be true, in general.

For arbitrary projectors $Q$ and $Q_{*}, Q A_{1}^{-1}=-A_{* 1}^{*-1} Q_{*}^{*}$. Indeed, premultiplication by $A_{* 1}^{*}$ and multiplication from the right by $A_{1}$ would yield $-Q_{*}^{*} B Q$ 
at the both sides. Next we have

$$
Q_{s}:=Q A_{1}^{-1} B=-A_{* 1}^{*-1}\left(Q_{*}^{*} B\right)=-A_{* 1}^{*-1}\left(-Q_{*}^{*} A_{* 1}^{*}\right)=A_{* 1}^{*-1} Q_{*}^{*} A_{* 1}^{*} .
$$

The latter expression does not depend on $Q$, that is why neither can $Q_{s}$. And vice versa, by the original definition $Q_{s}$ did not depend on specific choice of $Q_{*}$, that is why neither can $A_{* 1}^{*-1} Q_{*} A_{* 1}^{*}$. Further, $\operatorname{dim} \operatorname{Im} P_{*}=r$. We obtain directly

Lemma 2 The projectors

$$
Q_{s}=A_{* 1}^{*-1} Q_{*}^{*} A_{* 1}^{*}, \quad P_{s}=A_{* 1}^{*-1} P_{*}^{*} A_{* 1}^{*}
$$

do not depend on the specific choice of $Q$ or $Q_{*}$. Moreover, $\operatorname{dim} \operatorname{Im} P_{s}=r$ and $\operatorname{dim} \operatorname{Im} Q_{s}=m-r$.

From the above considerations it also follows that

$$
A_{1}^{*-1} Q^{*}=\left(Q A_{1}^{-1}\right)^{*}=-\left(A_{* 1}^{*-1} Q_{*}^{*}\right)^{*}=-Q_{*} A_{* 1}^{-1} \subseteq \operatorname{Ker} A^{*} .
$$

Thus, we can state

Corollary 1 Ker $A=\operatorname{Im} Q_{s}$ and $\operatorname{Ker} A^{*}=\operatorname{Im} Q_{* s}$.

In the next section we return to the complementary subspaces $\operatorname{Im} P_{s}$ and $\operatorname{Im} P_{* s}$.

When $A \in C\left(\mathcal{I}, L\left(\mathbb{C}^{m}, \mathbb{C}^{m}\right)\right)$, then there exist projectors $Q_{*}$ onto Ker $A^{*}$ and $P_{*}:=I-Q_{*}$, which are continuous. Indeed, the orthogonal projectors $\breve{Q}_{*}=I-\breve{P}_{*}, \breve{P}_{*}=A A^{+}$have this property by their definition. $\breve{P}_{*}$ projects onto $\operatorname{Im} A$. Thus, under assumption (T3) and (3) there exist $A_{1}, A_{* 1} \in$ $C\left(\mathcal{I}, L\left(\mathbb{C}^{m}, \mathbb{C}^{m}\right)\right)$ since $\breve{A}_{1}, \breve{A}_{* 1} \in C\left(\mathcal{I}, L\left(\mathbb{C}^{m}, \mathbb{C}^{m}\right)\right)$.

When $\phi \in C\left(\mathcal{I}, \mathbb{C}^{m}\right)$ is such that $A^{*} \phi \in C^{1}\left(\mathcal{I}, \mathbb{C}^{m}\right)$, we can split $\mathcal{L}^{*} \phi$ : $\mathcal{L}^{*} \phi=\left[\left(A^{*}\left(P_{* s} \phi\right)\right)^{\prime}-B^{*} P_{* s} \phi\right]-B^{*} Q_{* s} \phi$.

Now we use the following chain of identities:

$$
\begin{aligned}
& A_{1} Q_{s}=A_{1}\left[Q A_{1}^{-1}\left(A_{1}+B P\right)\right]=A_{1} Q A_{1}^{-1}\left(A+B-A P^{\prime} Q\right) \\
& =A_{1} Q P+A_{1} Q A_{1}^{-1} B-A_{1} Q P P^{\prime} Q=A_{1} Q A_{1}^{-1} B=Q_{* s}^{*} B .
\end{aligned}
$$

Therefore, the function $\phi_{2}:=Q_{* s} A_{1}^{*-1} s$ satisfies $B^{*} Q_{* s} \phi_{2}=B^{*} Q_{* s} A_{1}^{*-1} s=$ $Q_{s}^{*} s$. On the other hand, since $\operatorname{Im} P_{s}^{*}=\operatorname{Im} A_{* 1} P_{*} A_{* 1}^{-1}=\operatorname{Im} A^{*} A_{* 1}^{-1}=\operatorname{Im} P^{*}$, 
if the considerations leading us to (13) are taken into account, we can state that equation $\mathcal{L}^{*} \phi_{1}=P_{s}^{*} s$ has a solution $\phi_{1} \in \operatorname{Im} P_{* s}$. Summarizing the above for equation (13) with arbitrary $s \in C\left(\mathcal{I}, \mathbb{C}^{m}\right)$, with the decomposition $s=P_{s}^{*} s+Q_{s}^{*} s$, we are able to construct a solution $\phi:=\phi_{1}+\phi_{2}$. For this solution $\phi \in C\left(\mathcal{I}, \mathbb{C}^{m}\right)$ and $A^{*} \phi \in C^{1}\left(\mathcal{I}, \mathbb{C}^{m}\right)$ hold. Further we denote the function space

$$
C_{* A}^{1}\left(\mathcal{I}, \mathbb{C}^{m}\right):=\left\{\phi \in C\left(\mathcal{I}, \mathbb{C}^{m}\right): A^{*} \phi \in C^{1}\left(\mathcal{I}, \mathbb{C}^{m}\right)\right\}
$$

Remark 2 Clearly, the function space $C_{* A}^{1}\left(\mathcal{I}, \mathbb{C}^{m}\right)$ is by no means connected to any projector. We also must underline that the function spaces $C_{A^{*}}^{1}\left(\mathcal{I}, \mathbb{C}^{m}\right)$ and $C_{* A}^{1}\left(\mathcal{I}, \mathbb{C}^{m}\right)$ are different as the following example shows. When, however, $A \in C^{1}\left(\mathcal{I}, L\left(\mathbb{C}^{m}, \mathbb{C}^{m}\right)\right.$ holds, then $C_{A^{*}}^{1}\left(\mathcal{I}, \mathbb{C}^{m}\right)=C_{* A}^{1}\left(\mathcal{I}, \mathbb{C}^{m}\right)$.

Example: Given a positive scalar function $\alpha: \mathcal{I} \rightarrow \mathbb{R}$ that is Lipschitz but not continuously differentiable, $m=2, A(x)=\operatorname{diag}(\alpha(x), 0)$. We have $A(x)^{*}=A(x), P_{*}(x)=\operatorname{diag}(1,0)$. The function space $C_{A^{*}}^{1}$ contains all continuous functions $y: \mathcal{I} \rightarrow \mathbb{C}^{2}$ the first component $y_{1}$ of which is continuously differentiable. On the other hand, $y \in C_{* A}^{1}$ means that the first and second components are continuous but just the product $\alpha y_{1}$ is continuously differentiable. Hence constant functions belong to $C_{A^{*}}^{1}$ but they do not belong to $C_{* A}^{1}$. In the contrary, $C_{* A}^{1}$ contains the function $y_{1}=\frac{1}{\alpha}, y_{2}=0$ but $C_{A^{*}}^{1}$ does not.

Theorem 1 Under the assumptions (T1)-(T3), for arbitrary $\phi^{0} \in \mathbb{C}^{m}$ and $s \in C\left(\mathcal{I}, \mathbb{C}^{m}\right)$, there exists a solution $\phi \in C_{* A}^{1}\left(\mathcal{I}, C^{m}\right)$ of (13) such that

$$
A^{*}(\hat{x})\left(\phi(\hat{x})-\phi^{0}\right)=0
$$

holds.

Proof. Let

$$
\phi(\hat{x}):=P_{* s}(\hat{x}) \phi^{0}+Q_{* s}(\hat{x}) A_{1}^{*-1}(\hat{x}) s(\hat{x}) .
$$

Then (19) trivially holds. Let $w$ be the solution of the ODE $\mathcal{L}_{s}^{*} w=P_{s}^{*} s$ with initial value $w(\hat{x})=A_{1}^{*} \phi^{0}$. Then, $\phi_{1}:=P_{* s} A^{+*} P^{*} w$ solves the problem $\mathcal{L}^{*} \phi_{1}=P_{s}^{*} s$ and $\phi_{1}(\hat{x})=P_{* s} \phi^{0}$ takes place. A solution claimed in the Theorem is $\phi:=\phi_{1}+Q_{* s} A_{1}^{-T} s$. 
Definitions Equation of the form (13) is called adjoint equation of the DAE (2).

An initial value $\phi^{0} \in \mathbb{C}^{m}$ such that $Q_{* s}(\hat{x}) \phi^{0}=Q_{* s}(\hat{x}) A_{1}^{*-1}(\hat{x}) s(\hat{x})$ holds is called consistent with (13). Note that another transparent form of consistent initial value can be obtained if one uses the identity $Q_{* s} A_{1}^{*-1}=A_{* 1}^{-1} Q_{s}^{*}$.

Theorem 2 Under the assumptions (T1)-(T3), for arbitrary $\phi^{0} \in \mathbb{C}^{m}$ and $s \in C\left(\mathcal{I}, \mathbb{C}^{m}\right)$, the solution of the initial value problem (13),(19) is unique.

Proof. Let $\phi^{(1)}, \phi^{(2)}$ be a pair of different solutions. Due to linearity, $0=$ $\mathcal{L}^{*}\left(\phi^{(1)}-\phi^{(2)}\right)$ must hold. Multiply this identity by $Q^{*}$. Then,

$$
\begin{aligned}
& 0=-Q^{* \prime} A^{*}\left(\phi^{(1)}-\phi^{(2)}\right)-Q^{*} B^{*}\left(\phi^{(1)}-\phi^{(2)}\right) \\
& =-Q^{*} B_{0}^{*}\left(\phi^{(1)}-\phi^{(2)}\right)=-Q^{*} A_{1}^{*}\left(\phi^{(1)}-\phi^{(2)}\right)
\end{aligned}
$$

i.e., $A^{*}\left(\phi^{(1)}-\phi^{(2)}\right)=A^{*}\left(\phi^{(1)}-\phi^{(2)}\right)+Q^{*} A_{1}^{*}\left(\phi^{(1)}-\phi^{(2)}\right)=A_{1}^{*}\left(\phi^{(1)}-\phi^{(2)}\right)$. As a consequence, $A_{1}^{*}\left(\phi^{(1)}-\phi^{(2)}\right) \in C^{1}\left(\mathcal{I}, \mathbb{C}^{m}\right)$. Let $\psi:=A_{1}^{*}\left(\phi^{(1)}-\phi^{(2)}\right)$. We have

$\psi^{\prime}-B^{*} A_{1}^{*-1} \psi=0 \quad$ and $\quad \psi(\hat{x})=A^{*}\left(\psi^{(1)}(\hat{x})-\psi^{(2)}(\hat{x})\right)=A^{*}\left(\psi^{0}-\psi^{0}\right)=0$.

For $\psi$, however, (21) is a homogeneous ODE with zero initial value, thus, the only solution is $\psi=0$. Therefore, $\phi^{(1)}-\phi^{(2)}=A_{1}^{*-1} \psi=0$, in contrast to the assumption.

Accomplishing the section, we call attention to other representations of canonical projectors $P_{s}$ and $P_{* s}$. A reason for doing so is that the MoorePenrose inverse $A^{+}$is defined fully by $A$ alone, while our problem is characterized by (T1)-(T3). It turns out that other generalized inverses become useful to overcome this gap.

Let $P, P_{*}$ be arbitrary but fixed projectors as before and let $A_{1}, A_{* 1}$ be constructed by their use. It is an elementary exercise to check that both

$$
A_{r}(P):=P A_{1}^{-1} \quad \text { and } \quad A_{* r}\left(P_{*}\right):=\left(P_{*} A_{* 1}^{-1}\right)^{*}
$$

are reflexive generalized inverses of $A$. Indeed, $A_{r}(P) A A_{r}(P)=$ $\left(P A_{1}^{-1}\right) A\left(P A_{1}^{-1}\right)=P\left(A_{1}^{-1} A\right) P A_{1}^{-1}=P P P A_{1}^{-1}=P A_{1}^{-1}=A_{r}(P)$, $A A_{r}(P) A=A\left(P A_{1}^{-1}\right) A=A P\left(A_{1}^{-1} A\right)=A P P=A$; 


$$
\begin{aligned}
& A_{* r}\left(P_{*}\right) A A_{* r}\left(P_{*}\right)=\left(A_{* r}^{*}\left(P_{*}\right) A^{*} A_{* r}^{*}\left(P_{*}\right)\right)^{*}=\left(\left(P_{*} A_{* 1}^{-1}\right) A^{*}\left(P_{*} A_{* 1}^{-1}\right)\right)^{*} \\
& =\left(P_{*}\left(A_{* 1}^{-1} A^{*}\right) P_{*} A_{* 1}^{-1}\right)^{*}=\left(P_{*} P_{*} P_{*} A_{* 1}^{-1}\right)^{*}=\left(P_{*} A_{* 1}^{-1}\right)^{*}, \\
& \quad A A_{* r}\left(P_{*}\right) A=\left(A^{*} A_{* r}^{*}\left(P_{*}\right) A^{*}\right)^{*}=\left(A^{*}\left(P_{*} A_{* 1}^{-1}\right) A^{*}\right)^{*}=\left(A^{*} P_{*}\left(A_{* 1}^{-1} A^{*}\right)\right)^{*}= \\
& \left(A^{*} P_{*} P_{*}\right)^{*}=A^{* *}=A .
\end{aligned}
$$

By definition, for any reflexive inverse $A^{-}$of $A$, the products $A A^{-}$and $A^{-} A$ are projectors. In our case, when $A^{-}=A_{r}(P)$ or $A^{-}=A_{* r}\left(P_{*}\right)$ and we have

$$
A A_{r}(P)=A P A_{1}^{-1}=A A_{1}^{-1}=\left(A_{1}^{*-1} A^{*}\right)^{*}=P_{* s}^{*}, A_{r}(P) A=\left(P A_{1}^{-1}\right) A=
$$
$P\left(A_{1}^{-1} A\right)=P P=P$ and $A A_{* r}\left(P_{*}\right)=A\left(P_{*} A_{* 1}^{-1}\right)^{*}=\left(\left(P_{*} A_{* 1}^{-1}\right) A^{*}\right)^{*}=$ $\left(P_{*}\left(A_{* 1}^{-1} A^{*}\right)\right)^{*}=\left(P_{*} P_{*}\right)^{*}=P_{*}^{*}, A_{* r}\left(P_{*}\right) A=\left(P_{*} A_{* 1}^{-1}\right)^{*} A=\left(A^{*} P_{*} A_{* 1}^{-1}\right)^{*}=$ $\left(A^{*} A_{* 1}^{-1}\right)^{*}=A_{* 1}^{*-1} A=P_{s}$. The adjoint of the first identity and the latter one are worth remembering:

$$
P_{s}=A_{* r}\left(P_{*}\right) A \quad \text { and } \quad P_{* s}=A_{r}^{*}(P) A^{*},
$$

meantime recalling that neither $P_{s}$ nor $P_{* s}$ on the left hand side depend on $P$ or $P_{*}$ while the reflexive inverses on the right hand side do so. Finally, we add that the expression $P_{* s} u:=P_{* s} A^{+*} P^{*} w$ occuring above can be simplified as

$$
\begin{array}{r}
P_{* s} u:=P_{* s} A^{+*} P^{*} w=A_{r}^{*}(P) A^{*} A^{+*} P^{*} w=\left(P\left(A^{+} A\right) A_{r}(P)\right)^{*} w \\
=\left(P \breve{P} A_{r}(P)\right)^{*} w=\left((P \breve{P}) A_{r}(P)\right)^{*}=\left(P A_{r}(P)\right)^{*} w=\left(P P A_{1}^{-1}\right)^{*} w \\
=\left(P A_{1}^{-1}\right)^{*} w=A_{r}^{*}(P) w .
\end{array}
$$

\section{Fundamental solutions}

If Theorems 1 and 2 are applied to homogeneous equations $(s=0)$, then one can conclude that for each $\phi^{0} \in \operatorname{Im} P_{* s}(\hat{x})$ there exists a unique solution $\phi$ of

$$
\mathcal{L}^{*} \phi=0
$$

such that $\phi(\hat{x})=\phi^{0}$ and $\phi \in \operatorname{Im} P_{* s}$ hold. One also has that for all solutions of (24) the relation $\phi \in \operatorname{Im} P_{* s}$ holds. Thus, $S_{*}:=\operatorname{Im} P_{* s}$ is the solution space for $(24)$. We proved in previous section that $\operatorname{dim} S_{*}=r$. Due to (12), (14) and Corollary 1 ,

$$
\mathbb{C}^{m}=S_{*}(x) \oplus \operatorname{Ker} A^{*}(x)
$$


Meanwhile for the homogeneous equation

$$
\mathcal{L} y=0
$$

$Q_{s} y=0$, thus, the solution space is $S:=\operatorname{Im} P_{s}$. For an arbitrary $y^{0} \in \mathbb{C}^{m}$, the consistent initial value $y(\hat{x})$ at $x=\hat{x}$ satisfies $y(\hat{x})=P_{s}(\hat{x}) y^{0}$, or, equivalently, $A(\hat{x})\left(y(\hat{x})-y^{0}\right)=0$, or $P(\hat{x})\left(y(\hat{x})-y^{0}\right)=0$.

Remark 3 In [6]- where the coefficients were real - the relation $\mathbb{R}^{m}=$ $S(x) \oplus \operatorname{Ker} A(x)$ was obtained using geometrical arguments. Our result

$$
\mathbb{C}^{m}=S(x) \oplus \operatorname{Ker} A(x)
$$

comes directly from the representation (17).

Up to now, operators $\mathcal{L}$ and $\mathcal{L}^{*}$ were defined in $C_{A}^{1}\left(\mathcal{I}, \mathbb{C}^{m}\right)$ and $C_{* A}^{1}\left(\mathcal{I}, \mathbb{C}^{m}\right)$, only. Solutions also were considered in these spaces. Now we want to extend the definitions to handle matrix functions also. For this purposes, we introduce some definitions. First, for each $q$, we fix a basis in $\mathbb{C}^{q}$ and denote it by $\left\{e_{q}^{(1)}, \ldots, e_{q}^{(q)}\right\}: \operatorname{span}\left\{e_{q}^{(1)}, \ldots, e_{q}^{(q)}\right\}=\mathbb{C}^{q}$.

Definitions Let for integers $q$ and $\mathrm{s}$, the function $f_{q}^{s}: \underbrace{\mathbb{C}^{s} \otimes \ldots \otimes \mathbb{C}^{s}}_{q} \rightarrow$ $L\left(\mathbb{C}^{q}, \mathbb{C}^{s}\right)$ be defined implicitly so that for all $y^{(1)}, \ldots, y^{(q)} \in \mathbb{C}^{s}$ and $e_{q}^{(i)}$, $i=1, \ldots, q, f_{q}^{s}\left(y^{(1)}, \ldots, y^{(q)}\right) e_{q}^{(i)}=y^{(i)}$ hold.

With the notation $\mathbb{C}_{\mathcal{I}}^{s}:=\left\{y: \mathcal{I} \rightarrow \mathbb{C}^{s}\right\}$ and $L_{\mathcal{I}}^{q s}:=\left\{Y: \mathcal{I} \rightarrow L\left(\mathbb{C}^{q}, \mathbb{C}^{s}\right)\right\}$ let $F_{q}^{s}$ : $\underbrace{\mathbb{C}_{\mathcal{I}}^{s} \otimes \ldots \otimes \mathbb{C}_{\mathcal{I}}^{s}}_{q} \rightarrow L_{\mathcal{I}}^{q s}$ be defined implicitly so that for all $y^{(1)}, \ldots, y^{(q)} \in \mathbb{C}_{\mathcal{I}}^{s}$ and $e_{q}^{(i)}, i=1, \ldots, q$, and for all $x \in \mathcal{I}$

$$
F_{q}^{s}\left(y^{(1)}, \ldots, y^{(q)}\right)(x) e_{q}^{(i)}=f_{q}^{s}\left(y^{(1)}(x), \ldots, y^{(q)}(x)\right) e_{q}^{(i)}=y^{(i)}(x) .
$$

When $s=m$, the upper index $m$ will be omitted and we write shortly $f_{q}$ and $F_{q}$, respectively.

A set $\left\{y^{(1)}, \ldots, y^{(k)}\right\}$ of functions with $y^{(i)} \in C_{A}^{1}\left(\mathcal{I}, \mathbb{C}^{m}\right), i=1, \ldots, k$, will be called fundamental set of solutions of (26) if each $y^{(i)}$ is a solution of (26) and $\operatorname{span}\left\{y^{(1)}, \ldots, y^{(k)}\right\}=S$. In other words, $\operatorname{Im} F_{q}\left(y^{(1)}, \ldots, y^{(k)}\right)=S$.

Similarly, a set $\left\{\phi^{(1)}, \ldots, \phi^{(l)}\right\}$ of functions such that $\phi^{(i)} \in \mathbb{C}_{* A}^{1}\left(\mathcal{I}, \mathbb{C}^{m}\right)$, $i=1, \ldots, l$ will be called fundamental set of solutions of $(24)$ if each $\phi^{(i)}$ is a 
solution of $(24)$ and $\operatorname{span}\left\{\phi^{(1)}, \ldots, \phi^{(l)}\right\}=S_{*}$.

In other words, $\operatorname{Im} F_{q}\left(\phi^{(1)}, \ldots, \phi^{(l)}\right)=S_{*}$.

For brevity, a function $Y: \mathcal{I} \rightarrow L\left(\mathbb{C}^{k}, \mathbb{C}^{m}\right)$ is called fundamental solution of (26) if $Y=F_{k}\left(y^{(1)}, \ldots, y^{(k)}\right)$ for some fundamental set $\left\{y^{(1)}, \ldots, y^{(k)}\right\}$ of solutions of $(26)$.

Similarly, $\Phi: \mathcal{I} \rightarrow L\left(\mathbb{C}^{l}, \mathbb{C}^{m}\right)$ is called fundamental solution of (24) if $\Phi=F_{q}\left(\phi^{(1)}, \ldots, \phi^{(l)}\right)$ for some fundamental set $\left\{\phi^{(1)}, \ldots, \phi^{(l)}\right\}$ of solutions of (24).

When $Y$ and $\Phi$ are fundamental solutions, then $Y \in C_{A}^{1}\left(\mathcal{I}, L\left(\mathbb{C}^{k}, \mathbb{C}^{m}\right)\right)$ and $\Phi \in C_{* A}^{1}\left(\mathcal{I}, L\left(\mathbb{C}^{l}, \mathbb{C}^{m}\right)\right)$ where $C_{A}^{1}\left(\mathcal{I}, L\left(\mathbb{C}^{k}, \mathbb{C}^{m}\right)\right):=\left\{Y \in C\left(\mathcal{I}, L\left(\mathbb{C}^{k}, \mathbb{C}^{m}\right)\right):\right.$ $\left.P Y \in C^{1}\left(\mathcal{I}, L\left(\mathbb{C}^{k}, \mathbb{C}^{m}\right)\right)\right\}$ and $C_{* A}^{1}\left(\mathcal{I}, L\left(\mathbb{C}^{l}, \mathbb{C}^{m}\right)\right):=\left\{\Phi \in C\left(\mathcal{I}, L\left(\mathbb{C}^{l}, \mathbb{C}^{m}\right)\right):\right.$ $\left.A^{*} \Phi \in C^{1}\left(\mathcal{I}, L\left(\mathbb{C}^{l}, \mathbb{C}^{m}\right)\right)\right\}$, respectively, with corresponding $k$ and $l$.

Remark 4 Since $\operatorname{dim} S=\operatorname{dim} S_{*}=r$, for a fundamental set of solutions $k \geq r$ and $l \geq r$, respectively, must hold. In contrast to the regular explicit ODEs $(A=I)$, where $r=m$, and their adjoint equations, the fundamental sets of solutions are not linearly independent when $r<k \leq m, r<l \leq m$. From this point of view, it would be enough to define fundamental solutions only for $k=r$ and $l=r$. Then, for example, the values of Moore-Penrose inverse $Y^{+}$of a fundamental solution $Y$ would be easily computable, namely, $Y^{+}=\left(Y^{*} Y\right)^{-1} Y^{*}$. This restricted definition, however, would make some other computations complicated or impossible. The less computational inconvenience occurs if one uses $k=m$ and $l=m$. We will see below that there is a somewhat natural normalization and group properties also can be obtained.

Clearly, it is not reasonable to use fundamental solutions with $k>m$, $l>m$.

Definitions We call fundamental solutions $Y$ and $\Phi$ minimal if $k=r$ and $l=r$, respectively. Fundamental solutions $Y$ and $\Phi$ will be called maximal, if $k=m$ and $l=m$.

We remind that for $k \geq r$ the theoretical existence of fundamental solutions of (26) becomes trivial due to (27) and unique solvability of initial value problem. The construction using the scheme of the previous paragraph is as follows: Let $p_{k}^{(i)} \in C^{1}\left(\mathcal{I}, \mathbb{C}^{m}\right), i=1, \ldots, k$, be the solutions of the initial value problems for ODEs:

$$
L_{s} z=0, \quad z(\hat{x})=\hat{p}_{k}^{(i)},
$$


where $P \in \mathbb{C}^{1}\left(\mathcal{I}, L\left(\mathbb{C}^{m}, \mathbb{C}^{m}\right)\right.$ appearing in (6) is the complement of an arbitrary projector $Q$ onto Ker $A$ which satisfies (T3), $\hat{x} \in \mathcal{I}$ is arbitrary but fixed and the arbitrary but fixed set $\left\{\hat{p}_{k}^{(1)}, \ldots, \hat{p}_{k}^{(k)}\right\}$ is such that $\operatorname{span}\left\{\hat{p}_{k}^{(1)}, \ldots, \hat{p}_{k}^{(k)}\right\}=$ $\operatorname{Im} P(\hat{x})$. Then, together with an arbitrary projector $Q_{*} \in C\left(\mathcal{I}, L\left(\mathbb{C}^{m}, \mathbb{C}^{m}\right)\right)$ onto Ker $A^{*}$, we can construct $A_{1}, A_{* 1} \in C\left(\mathcal{I}, L\left(\mathbb{C}^{m}, \mathbb{C}^{m}\right)\right)$. Now,

$$
Y:=A_{* 1}^{*-1} A_{1} F_{k}\left(p_{k}^{(1)}, \ldots, p_{k}^{(k)}\right)
$$

is a fundamental solution.

For verification, first we recall a property mentioned after (4): if $\hat{p}_{k}^{(i)} \in$ $\operatorname{Im} P(\hat{x})$, and this is the case now, then $p_{k}^{(i)}=P p_{k}^{(i)}$. Consequently,

$$
F_{k}\left(p_{k}^{(1)}, \ldots, p_{k}^{(k)}\right)=P F_{k}\left(p_{k}^{(1)}, \ldots, p_{k}^{(k)}\right),
$$

yielding

$$
\begin{array}{r}
Y=A_{* 1}^{*-1} A_{1} P F_{k}\left(p_{k}^{(1)}, \ldots, p_{k}^{(k)}\right)=A_{* 1}^{*-1} A F_{k}\left(p_{k}^{(1)}, \ldots, p_{k}^{(k)}\right) \\
=A_{* 1}^{*-1} P_{*}^{*} A_{* 1}^{*} F_{k}\left(p_{k}^{(1)}, \ldots, p_{k}^{(k)}\right)=P_{s} F_{k}\left(p_{k}^{(1)}, \ldots, p_{k}^{(k)}\right) \subseteq \operatorname{Im} P_{s}=S .
\end{array}
$$

On the other hand,

$$
\begin{array}{r}
\operatorname{dim} \operatorname{Im} Y=\operatorname{dim} \operatorname{Im} F_{k}\left(p_{k}^{(1)}, \ldots, p_{k}^{(k)}\right) \\
=\operatorname{dim} \operatorname{Im} f_{k}\left(\hat{p}_{k}^{(1)}, \ldots, \hat{p}_{k}^{(k)}\right)=\operatorname{dim} \operatorname{Im} P(\hat{x})=r .
\end{array}
$$

In this chain first we used that $\operatorname{dim} \operatorname{Im} A_{* 1}^{*-1}=\operatorname{dim} \operatorname{Im} A_{1}=m$, then we exploited that $\mathcal{L}_{s}$ is an ordinary differential operator, finally we took the fact that $\left\{\hat{p}_{k}^{(i)}\right\}_{i=1}^{k}$ spans $\operatorname{Im} P(\hat{x})$ into account. This completes the check that $\operatorname{Im} Y=S$.

The above scheme requires the construction of the set $\left\{\hat{p}_{k}^{(i)}\right\}_{i=1}^{k}$ which might be inconvenient when $r \leq k<m$. When $k=m$ then, however, the above result also reads as follows:

Let $U$ be a fundamental solution of the regular ODE $\mathcal{L}_{s} z=0$ such that $U(\hat{x})=I$. Then, there exists a fundamental solution $Y$ of (26) such that $Y=$ $P_{s} U P(\hat{x})$. Indeed, we may set $\hat{p}_{m}^{(i)}=P(\hat{x}) e_{m}^{(i)}$, and since $U P(\hat{x})=P U P(\hat{x})$, one obtains $A_{* 1}^{*-1} A_{1} U P(\hat{x})=A_{* 1}^{*-1} A_{1} U P(\hat{x})=A_{* 1}^{*-1} A_{1} P U P(\hat{x})=P_{s} U P(\hat{x})$. It is worth to note that in a lot of cases, it is easy to construct $P(\hat{x})$.

Even when the dimension $k$ in the definition is fixed, the fundamental solution is not unique. It belongs to the basic knowledge that for the regular 
ODEs they may differ only by a constant invertible multiplier $M \in L\left(\mathbb{C}^{m}, \mathbb{C}^{m}\right)$ (from the right). First we derive here some important relationships between the fundamental solutions of the DAE (26) including the case when the dimensions differ. The same will be done for the adjoint equation (24). Then we turn to highlighting the connection between the fundamental solution pairs of (26) and (24).

Theorem 3 For any pair of minimal fundamental solutions $Y^{(1)}, Y^{(2)}$ there exists a $T \in L\left(\mathbb{C}^{r}, \mathbb{C}^{r}\right)$ such that $Y^{(2)}=Y^{(1)} T$ and $T$ is invertible.

The statement extends the result known for regular ODEs to DAEs.

Proof. It is enough to prove the statement for a pair $Y^{(1)}, Y^{(2)}$ where $Y^{(1)}$ is a minimal fundamental solution constructed above with a fixed $\hat{x} \in \mathcal{I}$ and a fixed basis $\left\{\hat{p}_{r}^{(i)}\right\}_{i=1}^{r}$ and $Y^{(2)}$ is an arbitrary minimal fundamental solution.

We may assume that for any $x \in \mathcal{I}, Y^{(1)}(x) \neq Y^{(2)}(x)$, otherwise, due to uniqueness, $Y^{(1)}=Y^{(2)}$ would hold and we could set $T=I$, where $I \in L\left(\mathbb{C}^{r}, \mathbb{C}^{r}\right)$ is the identity operator, which is clearly invertible.

On the other hand, by definition, $\operatorname{Im} Y^{(2)}(x)=S(x)=\operatorname{Im} Y^{(1)}(x)$, for $x \in \mathcal{I}$, so this holds at $x=\hat{x}$, too. Thus, with some $\left\{t^{(i)}\right\}_{i=1}^{r}, \hat{t}^{(i)} \in \mathbb{C}^{r}$, $i=1, \ldots, r$,

$$
Y^{(2)}(\hat{x}) e^{(i)}=A_{* 1}^{*-1}(\hat{x}) A_{1}(\hat{x}) f_{r}\left(\hat{p}_{r}^{(1)}, \ldots, \hat{p}_{r}^{(r)}\right) t^{(i)}, \quad i=1, \ldots, r,
$$

is valid, or simply, using the notation $\hat{P}:=f_{r}\left(\hat{p}_{r}^{(1)}, \ldots, \hat{p}_{r}^{(r)}\right)$ and $T:=$ $f_{r}\left(t^{(1)}, \ldots, t^{(r)}\right), Y^{(2)}(\hat{x})=A_{* 1}^{*-1}(\hat{x}) A_{1}(\hat{x}) \hat{P} T . \quad \operatorname{dim} \operatorname{Im} T=r$, otherwise $\operatorname{dim} \operatorname{Im} Y^{(2)}(\hat{x})$ would be less than $r$. Let us construct the minimal fundamental solution $Y$ by the previous receipt proceeding from $\hat{P} T$ instead of $\hat{P}$. Clearly, $Y(\hat{x})=Y^{(2)}(\hat{x})$ and therefore, by uniqueness theorem, $Y=Y^{(2)}$. On the other hand, $Y=Y^{(1)} T$ holds by construction.

Lemma 3 Let $Y \in C_{A}^{1}\left(\mathcal{I}, L\left(\mathbb{C}^{k}, \mathbb{C}^{m}\right)\right)$ be an arbitrary fundamental solution and $Y_{m}$ be an arbitrary minimal fundamental solution. Then, there exists a unique $W \in L\left(\mathbb{C}^{k}, \mathbb{C}^{r}\right)$ such that $Y=Y_{m} W$ and $\operatorname{dim} \operatorname{Im} W=r$.

Proof. With no restriction of the generality, we assume $Y_{m}=Y^{(1)}$ from Theorem 3. Then, by the same argument, $Y(\hat{x})=A_{* 1}^{*-1}(\hat{x}) A_{1}(\hat{x}) \hat{P} W, W \in$ $L\left(\mathbb{C}^{k}, \mathbb{C}^{r}\right)$ and $\operatorname{dim} \operatorname{Im} W=r$ must hold. The proof is accomplished if an auxiliary fundamental solution has been constructed proceeding from $\hat{P} W$ since it must coincide with $Y$. 
Lemma 4 Let $Y \in C_{A}^{1}\left(\mathcal{I}, L\left(\mathbb{C}^{k}, \mathbb{C}^{m}\right)\right)$ be an arbitrary fundamental solution and $Y_{m}$ be an arbitrary minimal fundamental solution. Then, there exists $V \in L\left(\mathbb{C}^{r}, \mathbb{C}^{k}\right)$ such that $Y_{m}=Y V$.

Proof. Let $Y=F_{k}\left(y^{(1)}, \ldots, y^{(k)}\right)$. Since $\operatorname{dim} \operatorname{Im} Y=r$, there exists a permutation $\mathcal{P}=\left\{i_{1}, \ldots, i_{k}\right\}$ such that $\left\{y^{\left(i_{1}\right)}, \ldots, y^{\left(i_{r}\right)}\right\}$ is a fundamental set of solutions of $(26)$ and therefore,$F_{r}\left(y^{\left(i_{1}\right)}, \ldots, y^{\left(i_{r}\right)}\right)$ is a minimal fundamental solution. By Theorem 3, there exists a unique $T \in L\left(\mathbb{C}^{r}, \mathbb{C}^{r}\right)$ such that $F_{r}\left(y^{\left(i_{1}\right)}, \ldots, y^{\left(i_{r}\right)}\right) T=Y_{m}$. Let $D \in L\left(\mathbb{C}^{r}, \mathbb{C}^{k}\right)$ defined by $D e_{r}^{(j)}=e_{k}^{\left(i_{j}\right)}$, $j=1, \ldots, r$, or more compactly, $D f_{r}^{r}\left(e_{r}^{(1)}, \ldots, e_{r}^{(r)}\right)=f_{r}^{k}\left(e_{k}^{\left(i_{1}\right)}, \ldots, e_{k}^{\left(i_{r}\right)}\right)$. Then, for all $x \in \mathcal{I}$,

$$
Y(x) D f_{r}^{r}\left(e_{r}^{(1)}, \ldots, e_{r}^{(r)}\right) T=f_{r}\left(y^{\left(i_{1}\right)}(x), \ldots, y^{\left(i_{r}\right)}(x)\right) T=Y_{m}(x) .
$$

Setting $V=D f_{r}^{r}\left(e_{r}^{(1)}, \ldots, e_{r}^{(r)}\right) T$, one arrives to the existence of a $V \in$ $L\left(\mathbb{C}^{k}, \mathbb{C}^{m}\right)$ claimed in the statement. Again, $Y_{m}$ cannot be fundamental, unless $\operatorname{dim} \operatorname{Im} V=r$ holds.

Remark 5 In this Lemma we could not claim uniqueness of $V$ as the following trivial example shows. Let $Y_{m}=F_{r}\left(y^{(1)}, \ldots, y^{(r)}\right), Y=F_{k}\left(y^{(1)}, \ldots, y^{(r)}\right.$, $0, \ldots, 0), k>r$. For any matrix $V$ the first $r$ rows of which form an identity block but the last $k-r$ rows are arbitrarely chosen, we have the relation $Y_{m}=Y V$.

Combining Lemmas 3 and 4 we arrive to the general

Theorem 4 For any pair of fundamental solutions $Y^{(1)} \in C_{A}^{1}\left(\mathcal{I}, L\left(\mathbb{C}^{k_{1}}, \mathrm{C}^{m}\right)\right)$ and $Y^{(2)} \in C_{A}^{1}\left(\mathcal{I}, L\left(\mathbb{C}^{k_{2}}, \mathbb{C}^{m}\right)\right)$, there exists $R \in L\left(\mathbb{C}^{k_{2}}, \mathbb{C}^{k_{1}}\right)$ such that $Y^{(2)}=$ $Y^{(1)} R$ and $\operatorname{dim} \operatorname{Im} R=r$.

Proof. Indeed, $Y^{(2)}=Y_{m} W=Y^{(1)} V W$, where $Y_{m}$ is an arbitrary minimal fundamental solution and $\mathrm{V}$ and $\mathrm{W}$ are chosen correspondingly by Lemmas. Let $R=V W . \operatorname{dim} \operatorname{Im} R=r$ holds otherwise $Y^{(2)}$ cannot be fundamental. $\square$

It is worth noting the special case $k_{1}=k_{2}=m$ :

Corollary 2 For any pair of maximal fundamental solutions $Y^{(1)}, Y^{(2)}$ there exists $R \in L\left(\mathbb{C}^{m}, \mathbb{C}^{m}\right)$ such that $Y^{(2)}=Y^{(1)} R$ and $\operatorname{dim} \operatorname{Im} R=r$. 
Based on decomposition (25) and Theorems 1 and 2, the existence of fundamental solutions $\Phi \in C_{* A}^{1}\left(\mathcal{I}, L\left(\mathbb{C}^{l}, \mathbb{C}^{m}\right), l=r, \ldots, m\right.$ can be obtained. Moreover, by analogous arguments as for the original homogeneous DAE, the analogues of Lemmas 3 and 4 and Theorems 3 and 4 hold for adjoint equations, just $Y$ must be changed for $\Phi$. An obvious change arises in the construction of fundamental solutions.

Let $p_{* l}^{(j)} \in C^{1}\left(\mathcal{I}, \mathbb{C}^{m}\right), i=1, \ldots, l$, be the solutions of the initial value problems for ODEs:

$$
L_{s}^{*} w=0, \quad w(\hat{x})=\hat{p}_{* l}^{(j)},
$$

where $P \in \mathbb{C}^{1}\left(\mathcal{I}, L\left(\mathbb{C}^{m}, \mathbb{C}^{m}\right)\right.$ appearing in (6) is the complement of an arbitrary projector $Q$ onto Ker $A$ which satisfies (T3), $\hat{x} \in \mathcal{I}$ is arbitrary but fixed and the arbitrary but fixed set $\left\{\hat{p}_{* l}^{(1)}, \ldots, \hat{p}_{* l}^{(l)}\right\}$ is such that $\operatorname{span}\left\{\hat{p}_{* l}^{(1)}, \ldots, \hat{p}_{* l}^{(l)}\right\}=$ $\operatorname{Im} P^{*}(\hat{x})$. Then, we can construct $A_{1} \in C\left(\mathcal{I}, L\left(\mathbb{C}^{m}, \mathbb{C}^{m}\right)\right)$. Now, let

$$
\Phi:=A_{1}^{*-1} P^{*} F_{l}\left(p_{* l}^{(1)}, \ldots, p_{* l}^{(l)}\right) \in C\left(\mathcal{I}, L\left(\mathbb{C}^{l}, \mathbb{C}^{m}\right)\right) .
$$

We obtain $A^{*} \Phi=P^{*} F_{l}\left(p_{* l}^{(1)}, \ldots, p_{* l}^{(l)}\right) \in C^{1}\left(\mathcal{I}, L\left(\mathbb{C}^{l}, \mathbb{C}^{m}\right)\right)$. Consequently, $\Phi \in$ $C_{* A}^{1}\left(\mathcal{I}, L\left(\mathbb{C}^{l}, \mathbb{C}^{m}\right)\right)$ and $\operatorname{Im} \Phi \subseteq \operatorname{Im} P_{* s}=S_{*}$. Further, $\Phi$ is a fundamental solution of (24). Indeed, $A_{1}^{*-1} P^{*}=P_{* s} A^{+*} P^{*}$ and due to (8)-(13), it involves that $A_{1}^{*-1} P^{*} p_{* l}^{(j)}=P_{* s} A^{+*} P^{*} p_{* l}^{(j)}, j=1, \ldots, l$ satisfies $(24)$. Noting that $P^{*} p_{* l}^{(j)}$ are solutions of the ODE $v^{\prime}-\left(P^{* \prime}+P^{*} B_{0}^{*} A_{1}^{*-1}\right) v=0$, the above choice of the initial values ensures

$$
\begin{array}{r}
\operatorname{dim} \operatorname{Im} \Phi=\operatorname{dim} \operatorname{Im} A_{1}^{*-1} P^{*} F_{l}\left(p_{* l}^{(1)}, \ldots, p_{* l}^{(l)}\right) \\
=\operatorname{dim} \operatorname{Im} P^{*} F_{l}\left(p_{* l}^{(1)}, \ldots, p_{* l}^{(l)}\right)=\operatorname{dim} \operatorname{Im} F_{l}\left(P^{*} p_{* l}^{(1)}, \ldots, P^{*} p_{* l}^{(l)}\right) \\
=\operatorname{dim} \operatorname{Im} f_{l}\left(P^{*} \hat{p}_{* l}^{(1)}, \ldots, P^{*} \hat{p}_{* l}^{(l)}\right)=\operatorname{dim} \operatorname{Im} P^{*}=r
\end{array}
$$

This completes the check that $\operatorname{Im} \Phi=S_{*}$. Now, the verification of the analogues of the statements about relationships between different fundamental solutions of the adjoint equations becomes trivial if one follows the scheme of the corresponding proofs for the original equation.

Now we state a connection between fundamental solutions of (26) and (24).

Theorem 5 Let $Y \in C_{A}^{1}\left(\mathcal{I}, L\left(\mathbb{C}^{k}, \mathbb{C}^{m}\right)\right)$ and $\Phi \in C_{* A}^{1}\left(\mathcal{I}, L\left(\mathbb{C}^{l}, \mathbb{C}^{m}\right)\right)$ be an arbitrary pair of fundamental solutions for (26) and (24). Then,

$$
\left(\Phi^{*} A Y\right)^{\prime}=0
$$


or, in other words, there exists $\Pi \in L\left(\mathbb{C}^{l}, \mathbb{C}^{k}\right)$ such that for all $x \in \mathcal{I}$, $(\Phi(x))^{*} A(x) Y(x)=\Pi$ holds.

Remark 6 When $A(x)=I$, this result is the well-known Lagrange identity for regular ODE-s.

Proof.

$$
\begin{array}{r}
\left(\Phi^{*} A Y\right)^{\prime}=\left(\Phi^{*} A P Y\right)^{\prime}=\left(\Phi^{*} A\right)^{\prime} P Y+\Phi^{*} A(P Y)^{\prime} \\
=\Phi^{*} B P Y-\Phi^{*} A\left(P A_{1}^{-1} B_{0}-P^{\prime}\right) P Y=\Phi^{*}\left(B-A P A_{1}^{-1} B_{0}\right) P Y \\
=\Phi^{*}\left(I-A A_{1}^{-1}\right) B P Y=\Phi^{*}\left(I-P_{* s}^{*}\right) B P Y=\left(\Phi^{*} Q_{* s}^{*}\right) B P Y=0 .
\end{array}
$$

In contrast to the regular ODEs, when minimal and maximal fundamental solutions are of the same dimensions, and thus, they are invertible, for DAEs this is not the case. One may ask about the relation of a generalized inverse of a fundamental solution for the DAE to the adjoint equation. Clearly, these results are closely related to the derivation of the adjoint equation described in the previous paragraph. First we concern maximal fundamental solutions with a basic value at a fixed point.

Definition A maximal fundamental solution $Y$ of (26) will be called normalized at $\hat{x}$ if $A(\hat{x})(Y(\hat{x})-I)=0$. A maximal fundamental solution $\Phi$ of (24) will be called normalized at $\hat{x}$ if $A^{*}(\hat{x})(\Phi(\hat{x})-I)=0$.

By virtue of (17) and (12), we immediately obtain $P_{s}(\hat{x}) Y(\hat{x})=P_{s}(\hat{x})$ and $P_{* s}(\hat{x}) \Phi(\hat{x})=P_{* s}(\hat{x})$ for maximal fundamental solutions normalized at $\hat{x}$. Thus, solutions of the initial value problems posed with initial values $Y(\hat{x})=P_{s}(\hat{x})$ and $\Phi(\hat{x})=P_{* s}(\hat{x})$ provide to us a pair of such $Y$ and $\Phi$. The simplest consequence is that for each $\hat{x}$, there exist both maximal fundamental solutions normalized at this $\hat{x}$. Further, we can state

Corollary 3 Provided $Y$ and $\Phi$ are maximal fundamental solutions of (26) and (24) normalized at the same point $\hat{x}$, relation $\Phi^{*}(\hat{x}) A(\hat{x})=A(\hat{x}) Y(\hat{x})$ is valid. For them, Theorem 5 yields $\Pi \in L\left(\mathbb{C}^{m}, \mathbb{C}^{m}\right), \Pi=A(\hat{x})$.

Lemma 5 For each $\hat{x}$, both maximal fundamental solutions $Y$ and $\Phi$ normalized at $\hat{x}$ are unique. 
Proof. Let $Y$ be a maximal fundamental solution of (26) and $U$ be the fundamental solution of ODE $\mathcal{L}_{s} z=0$ such that $U(\hat{x})=I$. By definition, $\mathcal{L}_{s}\left(P Y e_{i}\right)=0, i=1, \ldots, m$ and we showed that $P U P(\hat{x})=U P(\hat{x})$. Since $Q_{s} Y=0$, we have $Y=P_{s} Y=P_{s} P Y=P_{s} U P(\hat{x}) \mathcal{N}$ with some $\mathcal{N} \in L\left(\mathbb{C}^{m}, \mathbb{C}^{m}\right)$. Since $Y$ is assumed to be normalized at $\hat{x}$, we obtain $P_{s}(\hat{x}) \mathcal{N}=P_{s}(\hat{x})$. Now, taking into account that $P_{s}(\hat{x})=A_{* 1}^{*-1}(\hat{x}) A_{1}(\hat{x}) P(\hat{x})$ with arbitrary $P$ and $P_{*}$, we arrive at $P(\hat{x}) \mathcal{N}=P(\hat{x})$, i.e., $Y=P_{s} U P(\hat{x})$.

Let $W$ be the fundamental solution of ODE $\mathcal{L}_{s}^{*} w=0$ such that $W(\hat{x})=I$. We checked that $P^{*} W=P^{*} W P^{*}(\hat{x})$ and $\mathcal{L}^{*}\left(P_{* s} A^{+*} P^{*}\left(W e_{i}\right)\right)=0$. Noting that

$$
\begin{array}{r}
P_{* s} A^{+*} P^{*} W=A_{1}^{*-1} A^{*} A^{+*} P^{*} W \\
=A_{1}^{*-1} \breve{P} P^{*} W=A_{1}^{*-1} P^{*} W=A_{1}^{*-1} P^{*} W P^{*}(\hat{x}),
\end{array}
$$

we can state that any maximal fundamental solution of (24) is of the form $\Phi=$ $A_{1}^{*-1} P^{*} W P^{*}(\hat{x}) \mathcal{M}$ with some $\mathcal{M} \in L\left(\mathbb{C}^{m}, \mathbb{C}^{m}\right)$. Since $P_{* s}(\hat{x})=A_{1}^{*-1} A^{*}(\hat{x})$, the condition of the normalization yields now $P^{*}(\hat{x}) \mathcal{M}=A^{*}(\hat{x})$, i.e. $\Phi=$ $A_{1}^{*-1} P^{*} W A^{*}(\hat{x})=A_{r}^{*}(P) W A^{*}(\hat{x})$.

We underline again that in spite of their form obtained above, both $Y$ and $\Phi$ are independent of $P$ and $P_{*}$ since the initial values $P_{s}(\hat{x})$ and $P_{* s}(\hat{x})$ do not depend on them.

We recall now that $W=U^{*-1}$. In order to get the analogue for the pair of maximal fundamental solutions $Y$ and $\Phi$ normalized at the same $\hat{x}$, we introduce the proper generalized inverses.

Let $Y^{-}$be the reflexive inverse of $Y$ such that $Y Y^{-}=P_{s}$ and $Y^{-} Y=$ $P_{s}(\hat{x})$, while $\Phi^{-}$be the reflexive inverse of $\Phi$ such that $\Phi \Phi^{-}=P_{* s}$ and $\Phi^{-} \Phi=P_{* s}(\hat{x})$. One checks that they can be given explicitly, namely, $Y^{-}=P_{s}(\hat{x}) U^{-1} P$ and $\Phi^{-}=A_{r}^{*}(P)(\hat{x}) W^{-1} A^{*}$. Since the reflexive generalized inverses with the prescibed products are unique and neither $Y$ nor $\Phi$ depend on $P$ or $P_{*}, Y^{-}$and $\Phi^{-}$are independent of them, as well.

Clearly, $Y^{-*}$ and $\Phi^{-*}$ are reflexive inverses of $Y^{*}$ and $\Phi^{*}$, respectively. Note, however, that for these reflexive inverses $Y^{*-}:=Y^{-*}$ and $\Phi^{*-}:=\Phi^{-*}$ one has

$$
Y^{*} Y^{*-}=P_{s}^{*}(\hat{x}), Y^{*-} Y^{*}=P_{s}^{*}, \quad \text { and } \quad \Phi^{*} \Phi^{*-}=P_{* s}^{*}(\hat{x}), \Phi^{*-} \Phi^{*}=P_{* s}^{*} .
$$

In the next Theorem we use $Y^{-}, Y^{*-}, \Phi^{-}, \Phi^{*-}$ defined in this way. 
Theorem 6 Given the maximal fundamental solution $Y$ of (26) normalized at $\hat{x}$, then the maximal fundamental solution of (24) normalized at $\hat{x}$ is

$$
\Phi:=A_{r}^{*}(P) Y^{*-} A^{*}(\hat{x}) .
$$

Given the maximal fundamental solution $\Phi$ of (24) normalized at $\hat{x}$, then the maximal fundamental solution of (26) normalized at $\hat{x}$ is

$$
Y:=A_{* r}\left(P_{*}\right) \Phi^{*-} A(\hat{x}) .
$$

Proof. By Lemma $5, \Phi=A_{r}^{*}(P) W A^{*}(\hat{x})$. We also use $A_{r}^{*}(P)=A_{r}^{*}(P) P^{*}$ and $A=A P_{s}$ and the explicit form of the inverse $Y^{-}$and get

$$
\begin{gathered}
\Phi=A_{r}^{*}(P) W A^{*}(\hat{x})=\left(A_{r}^{*}(P) P^{*}\right) U^{*-1}\left(A(\hat{x}) P_{s}(\hat{x})\right)^{*} \\
=A_{r}^{*}(P)\left(P^{*} U^{*-1} P_{s}(\hat{x})^{*}\right) A^{*}(\hat{x})=A_{r}^{*}(P) Y^{*-} A^{*}(\hat{x}) .
\end{gathered}
$$

For the second part, above $Y=P_{s} U P(\hat{x})$ obtained in Lemma 5, we use $A_{* r}\left(P_{*}\right) A=P_{s}$ and $A_{r}(P) A=P$. Then, using the explicit form of the inverse $\Phi^{-}$,

$$
\begin{array}{r}
Y=P_{s} U P(\hat{x})=\left(A_{* r}\left(P_{*}\right) A\right) W^{*-1}\left(A_{r}(P)(\hat{x}) A(\hat{x})\right) \\
=A_{* r}\left(P_{*}\right)\left(A W^{*-1}\left(A_{r}(P)(\hat{x})\right) A(\hat{x})\right)=A_{* r}\left(P_{*}\right) \Phi^{*-} A(\hat{x}) .
\end{array}
$$

Among the fundamental solutions the maximal ones stand out by a computational comfort that is quite similar to the regular ODE case. This is due to the natural normalization used above. What concerns minimal fundamental solutions, so there seems to be no canonical way to normalize since there is no distinguished basis of $\operatorname{Im} P_{s}(\hat{x})$ to start with.

Let us finish this section by realizing the wanted group properties of maximal fundamental solutions $Y(., \hat{x}), \Phi(., \hat{x})$ of (26) and (24) normalized at $\hat{x}$.

Theorem 7 For normalized maximal fundamental solutions of (26) and (24), it holds that

$$
\begin{array}{r}
Y(x, \hat{x})^{-}=Y(\hat{x}, x), \quad \Phi(x, \hat{x})^{-}=\Phi(\hat{x}, x), \\
Y(x, \hat{x})=Y(x, z) Y(z, \hat{x}), \quad \Phi(x, \hat{x})=\Phi(x, z) \Phi(z, \hat{x}),
\end{array}
$$

for all $x, \hat{x}, z \in \mathcal{I}$. 
Proof. From above we have the representations

$$
\begin{aligned}
& Y(x, \hat{x})=P_{s}(x) U(x, \hat{x}) P(\hat{x}), \\
& Y(x, \hat{x})^{-}=P_{s}(\hat{x}) U(\hat{x}, x) P(x), \\
& \Phi(x, \hat{x})=A_{r}^{*}(P)(x) W(x, \hat{x}) A^{*}(\hat{x}), \\
& \Phi(x, \hat{x})^{-}=A_{r}^{*}(P)(\hat{x}) W(\hat{x}, x) A^{*}(x) .
\end{aligned}
$$

Hence, the first pair of relations is obvious. Further, compute

$$
\begin{aligned}
& Y(x, z) Y(z, \hat{x})=P_{s}(x) U(x, z) P(z) P_{s}(z) U(z, \hat{x}) P(\hat{x}) \\
&=P_{s}(x) U(x, z) P(z) U(z, \hat{x}) P(\hat{x}) \\
&=P_{s}(x) U(x, z) U(z, \hat{x}) P(\hat{x}) \\
&=P_{s}(x) U(x, \hat{x}) P(\hat{x})=Y(x, \hat{x}) . \\
& \Phi(x, z) \Phi(z, \hat{x})=A_{r}^{*}(P)(x) W(x, z) A^{*}(z) A_{r}^{*}(P)(z) W(z, \hat{x}) A^{*}(\hat{x}) \\
&=A_{r}^{*}(P)(x) W(x, z) P^{*}(z) W(z, \hat{x}) A^{*}(\hat{x}) \\
&=\left(A_{r}^{*}(P)(x) P^{*}(x)\right) U^{*}(z, x) P^{*}(z) U^{*}(\hat{x}, z) A^{*}(\hat{x}) \\
&=A_{r}^{*}(P)(x)(P(z) U(z, x) P(x))^{*} U^{*}(\hat{x}, z) A^{*}(\hat{x}) \\
&=A_{r}^{*}(P)(x) P^{*}(x) U^{*}(z, x) U^{*}(\hat{x}, z) A^{*}(\hat{x}) \\
&=A_{r}^{*}(P)(x) P^{*}(x) U^{*}(\hat{x}, x) A^{*}(\hat{x}) \\
&=A_{r}^{*}(P)(x) W(x, \hat{x}) A^{*}(\hat{x})=\Phi(x, \hat{x}) .
\end{aligned}
$$

For the normalized maximal fundamental solution $Y(x, \hat{x})$ and each arbitrary fundamental solution $\tilde{Y}(x)$ we have the indentity

$$
\tilde{Y}(x)=Y(x, \hat{x}) \tilde{Y}(\hat{x}) .
$$

Supposed $\tilde{Y}(x)$ is minimal and the columns of $\tilde{Y}(\hat{x})$ form an orthogonal basis of the subspace $S(\hat{x})$, the relation

$$
\tilde{Y}(\hat{x})^{*} \tilde{Y}(\hat{x})=I
$$

becomes true while $\tilde{Y}(\hat{x}) \tilde{Y}(\hat{x})^{*}$ represents the orthoprojector of $\mathbb{C}^{m}$ onto $S(\hat{x})$. Then, due to $\tilde{Y}(\hat{x}) \tilde{Y}(\hat{x})^{*} P_{s}(\hat{x})=P_{s}(\hat{x}), Y(x, \hat{x}) P_{s}(\hat{x})=Y(x, \hat{x})$, multiplying the indentity $(40)$ by $\tilde{Y}(\hat{x})^{*} P_{s}(\hat{x})$ leads to

$$
\tilde{Y}(x) \tilde{Y}(\hat{x})^{*} P_{s}(\hat{x})=Y(x, \hat{x}) .
$$

If we start with two different orthogonal basic systems of $S(\hat{x})$ we obtain two different minimal fundamental solutions but both of them satisfy (41) and (42). Therefore, thinking of normalizing minimal fundamental solutions via (41) makes no sense. A similar argument is true for the minimal fundamental solutions of the adjoint equation (24). 


\section{Index definition for adjoint equation}

Under the only assumptions (T1)-(T3), equation (13) is not a DAE in standard form. We will show now that we can extend the definition of DAE with index 1 to include (13).

Consider equations of the form

$$
\left(A^{*} \varphi\right)^{\prime}-B^{*} \varphi=p
$$

where $A, B: \mathcal{I} \rightarrow L\left(\mathbb{C}^{m}, \mathbb{C}^{m}\right)$ are continuous matrix functions that satisfy assumptions (T1)-(T3). Note that in terms of the adjoint matrices these assumptions are the same as

(T1) $\operatorname{dim} \operatorname{Im} A(x)^{*} \equiv r<m$ for all $x \in \mathcal{I}$

(T2) the matrix pencil $\left\{A(x)^{*}, B(x)^{*}\right\}$ is regular and has index 1 for $x \in \mathcal{I}$,

(T3) there exists a projector $Q^{*} \in C^{1}\left(\mathcal{I}, L\left(\mathbb{C}^{m}, \mathbb{C}^{m}\right)\right.$ such that $\operatorname{Im} A(x)^{*}=$ $\operatorname{Ker} Q(x)^{*}$ for $x \in \mathcal{I}$.

With $P^{*}=I-Q^{*}, P^{*} A^{*}=A^{*}$ we derive $\left(A^{*} \varphi\right)^{\prime}=\left(P^{*} A^{*} \varphi\right)^{\prime}=P^{*}\left(A^{*} \varphi\right)^{\prime}+$ $P^{*^{\prime}} A \varphi$ for all $\varphi \in C_{*_{A}}^{1}\left(\mathcal{I}, \mathbb{C}^{m}\right)$. Recall that this function space contains all continuous functions $\varphi$ which have a continuously differentiable part $A^{*} \varphi$. By means of this expression we rewrite (43) as

$$
P^{*}\left(A^{*} \varphi\right)^{\prime}+P^{*^{\prime}} A \varphi-B^{*} \varphi=p .
$$

Trivially, if $\varphi \in C_{*_{A}}^{1}$ solves (44), then the pair $\varphi, w, w:=A^{*} \varphi$ represents a solution of the enlarged system

$$
\begin{gathered}
P^{*} w^{\prime}+P^{*^{\prime}} w-B^{*} \varphi=p, \\
w-A^{*} \varphi=0 .
\end{gathered}
$$

Written in compact form the system (45), (46) is simply

$$
\left(\begin{array}{ll}
P^{*} & 0 \\
0 & 0
\end{array}\right)\left(\begin{array}{c}
w \\
\varphi
\end{array}\right)^{\prime}+\left(\begin{array}{cc}
P^{*^{\prime}} & -B^{*} \\
I & -A^{*}
\end{array}\right)\left(\begin{array}{l}
w \\
\varphi
\end{array}\right)=\left(\begin{array}{l}
p \\
0
\end{array}\right) .
$$


Theorem 8 The coefficients of (47) are continuous and satisfy (T1)- (T3) correspondingly.

Proof. (T1) and (T3) are given trivially. It remains to check whether the matrix

$$
G:=\left(\begin{array}{ll}
P^{*} & 0 \\
0 & 0
\end{array}\right)+\left(\begin{array}{cc}
P^{*^{\prime}} & -B^{*} \\
I & -A^{*}
\end{array}\right)\left(\begin{array}{cc}
Q^{*} & 0 \\
0 & I
\end{array}\right)=\left(\begin{array}{cc}
P^{*}+P^{*^{\prime}} Q^{*} & -B^{*} \\
Q^{*} & -A^{*}
\end{array}\right)
$$

remains nonsingular. In fact, $G(x) z=0$, means (we drop the argument $x$ )

$$
Q^{*} z_{1}-A^{*} z_{2}=0, \quad P^{*} z_{1}+P^{*^{\prime}} Q^{*} z_{1}-B^{*} z_{2}=0,
$$

what implies $Q^{*} z_{1}=0, A^{*} z_{2}=0, P^{*} z_{1}-B^{*} z_{2}=0$, i. e. $Q^{*} z_{1}=0, z_{2} \in \operatorname{Ker} A^{*}, B^{*} z_{2}=P^{*} z_{1} \in \operatorname{Im} A^{*}$.

Due to the index-1-property of $\left\{A^{*}, B^{*}\right\}$ we find $z_{2}=0$, thus $z_{1}=0$.

It comes out that the enlarged system (45), (46) resp. (47) is an index-1 DAE in standard form. Due to the respective theory, for arbitrary continuous right hand sides, there are continuous solutions $w, \varphi$ that have continuously differentiable components $P^{*} w$. In particular, for continuous $p$, equation (47) has those solutions, but due to (46) we have

$$
A^{*} \varphi=P^{*} A^{*} \varphi=P^{*} w,
$$

what means that $\varphi$ belongs to $C_{*_{A}}^{1}$. In this sense, (43) and the standard form DAE (47) are equivalent. This gives rise to define equation (43) to be index-1 tractable if (T1)-(T3) are satisfied.

By this, the original DAE (2) is index-1 tractable if and only if (43) is so.

It should be mentioned that the enlarged system

$$
\begin{gathered}
w^{\prime}-B^{*} \varphi=p, \\
w-A^{*} \varphi=0
\end{gathered}
$$

instead of (47) will not do, since this DAE that has also standard form is of higher index. Namely, the respective matrix

$$
\left(\begin{array}{cc}
I & B^{*} \\
0 & -A^{*}
\end{array}\right)
$$

becomes nonsingular. 


\section{Acknowledgement}

The manuscript of this work was prepared basically during the visits of the first author at the Department of Numerical Methods, Institute of Applied Mathematics at Humboldt University. She is grateful for this possibility to all persons there arranging these visits.

\section{References}

[1] A. A. Abramov: On the transfer of boundary conditions for systems of linear ordinary differential equations. Zh. Vychisl. Mat. Mat. Fiz. Vol. 1, No. 3, pp. 542-545 (in Russian; English transl. in: USSR J. Comp. Math. Math. Phys.) (1961)

[2] K. Balla: Linear subspaces for linear DAEs of index 1. Computers Math. Applic. Vol. 32, No. 4/5, pp. 81-86 (1996)

[3] K. Balla: Boundary conditions and their transfer for differentialalgebraic equations of index 1 Computers Math. Applic. Vol. 31, No. 10, pp. 1-5 (1996)

[4] K. Balla, R. März: Transfer of boundary conditions for DAEs of index 1. SIAM J. Numer. Anal. Vol. 33, No. 6, pp. 2318-2332 (1996)

[5] E. A. Coddington, N. Levinson: Theory of ordinary differential equations. Mc Graw Hill, New York, 1955.

[6] E. Griepentrog, R. März: Differential-Algebraic Equations and Their Numerical Treatment Leipzig, Teubner Verlag, 1986.

[7] R. März: Extra-ordinary differential equations. Attempts to an analysis of differential-algebraic systems.In: European Congress of Mathematics, Budapest, July 22-26, 1996, Vol. 1. (eds.: A. Balog, G. O. H. Katona, A. Recski, D. Szász). Series "Progress in Mathematics" Vol. 168. Birkhäuser Verlag, pp. 313-334, 1998.

[8] R. März: Numerical methods for differential-algebraic equations Acta Numerica, pp. 141-198 (1992) 